\title{
Constructing simplicial branched covers
}

\author{
Nikolaus Witte* \\ (Communicated by T. Grundhöfer)
}

\begin{abstract}
Izmestiev and Joswig described how to obtain a simplicial covering space (the partial unfolding) of a given simplicial complex, thus obtaining a simplicial branched cover [Adv. Geom. $3: 191-255,2003]$. We present a large class of branched covers which can be constructed via the partial unfolding. In particular, for $d \leq 4$ every closed oriented PL $d$-manifold is the partial unfolding of some polytopal $d$-sphere.
\end{abstract}

Key words. Geometric topology, construction of combinatorial manifolds, branched covers.

2000 Mathematics Subject Classification. 57M12, 57Q99, 05C15, 57M25

\section{Introduction}

Branched covers are applied frequently in topology - most prominently in the study, construction and classification of closed oriented PL $d$-manifolds. First results are by Alexander [1] in 1920, who observed that any closed oriented PL $d$-manifold $M$ is a branched cover of the $d$-sphere. Unfortunately Alexander's proof does not allow for any (reasonable) control over the number of sheets of the branched cover, nor over the topology of the branching set: The number of sheets depends on the size of some triangulation of $M$ and the branching set is the codimension 2-skeleton of the $d$-simplex.

However, in dimension $d \leq 4$, the situation is fairly well understood. By results of Hilden [8] and Montesinos [17] any closed oriented 3-manifold $M$ arises as 3-fold simple branched cover of the 3 -sphere branched over a link. In dimension four the situation becomes increasingly difficult. First Piergallini [21] showed how to obtain any closed oriented PL 4-manifold as a 4-fold branched cover of the 4-sphere branched over a transversally immersed PL-surface [21]. Iori \& Piergallini [11] then improved the standing result showing that the branching set may be realized locally flat if one allows for a fifth sheet for the branched cover, thus proving a long-standing conjecture by Montesinos [18]. The

\footnotetext{
*The authors is supported by Deutsche Forschungsgemeinschaft, DFG Research Group "Polyhedral Surfaces".
} 
question as to whether any closed oriented PL 4-manifold can be obtained as 4-fold cover of the 4-sphere branched over a locally flat PL-surface is still open.

For the partial unfolding and the construction of closed oriented combinatorial 3manifolds we recommend Izmestiev \& Joswig [14]. Their construction has recently been simplified significantly by Hilden, Montesinos, Tejada \& Toro [9]. For those able to read German additional analysis and examples can be found in [24]. The partial unfolding is implemented in the software package polymake [6].

This work has been greatly inspired by a paper of Hilden, Montesinos, Tejada \& Toro [9] and their bold approach. However, the techniques developed in the following turn out to differ substantially from the ideas in [9], allowing for stronger results in dimension three and generalization to arbitrary dimensions.

Outline of the paper. After some basic definitions and notations the partial unfolding $\widehat{K}$ of a simplicial complex $K$ is introduced. The partial unfolding defines a projection $p: \widehat{K} \rightarrow K$ which is a simplicial branched cover if $K$ meets certain connectivity assumptions. We define combinatorial models of key features of a branched cover, namely the branching set and the monodromy homomorphism.

Sections 2 and 3 are related, yet self-contained. The main result of this paper is presented in Theorem 2.1, and we give an explicit construction of a combinatorial $d$ sphere $S$ such that $p: \widehat{S} \rightarrow S$ is equivalent to a given simple, $(d+1)$-fold branched cover $r: X \rightarrow \mathbb{S}^{d}$ (with some additional restriction for the branching set of $r$ ). Theorem 2.1 is then applied to the construction of closed oriented PL $d$-manifolds as branched covers for $d \leq 4$. The construction of $S$ and the proof of its correctness take up the entire Section 2 .

Finally, in Section 3 we discuss how to extend a $k$-coloring of a subcomplex $L \subset$ $K$ of a simplicial $d$-complex $K$ to a $\max \{k, d+1\}$-coloring of a refinement $K^{\prime}$ of $K$ such that $L$ is again a subcomplex of $K^{\prime}$. Since $K^{\prime}$ is constructed from $K$ via finitely many stellar subdivisions of edges, all properties invariant under these subdivisions are preserved, e.g. polytopality, regularity, shellability, and others. This improves an earlier result by Izmestiev [12].

1.1 Basic definitions and notations. A simplicial complex $K$ is a combinatorial $d$-sphere or combinatorial $d$-ball if it is piecewise linear homeomorphic to the boundary of the $(d+1)$-simplex, respectively to the $d$-simplex. Equivalently, $K$ is a combinatorial $d$-sphere or $d$-ball if there is a common refinement of $K$ and the boundary of the $(d+1)$ simplex, respectively the $d$-simplex. A simplicial complex $K$ is a combinatorial manifold if the vertex link of each vertex of $K$ is a combinatorial sphere or a combinatorial ball. A manifold $M$ is PL if and only if $M$ has a triangulation as a combinatorial manifold. For an introduction to PL-topology see Björner [2, Part II], Hudson [10], and Rourke \& Sanderson [22].

A finite simplicial complex is pure if all the inclusion maximal faces, called the facets, have the same dimension. We call a codimension 1-face of a pure simplicial complex $K \mathrm{a}$ ridge, and the dual graph $\Gamma^{*}(K)$ of $K$ has the facets as its node set, and two nodes are adjacent if they share a ridge. We denote the 1-skeleton of $K$ by $\Gamma(K)$, its graph.

Further it is often necessary to restrict ourselves to simplicial complexes with certain connectivity properties: A pure simplicial complex $K$ is strongly connected if its 
dual graph $\Gamma^{*}(K)$ is connected, and locally strongly connected if the $\operatorname{star} \operatorname{st}_{K}(f)$ of $f$ is strongly connected for each face $f \in K$. If $K$ is locally strongly connected, then connected and strongly connected coincide. Further we call $K$ locally strongly simply connected if for each face $f \in K$ with codimension $\geq 2$ the link $\operatorname{lk}_{K}(f)$ of $f$ is simply connected, and finally, $K$ is nice if it is locally strongly connected and locally strongly simply connected. Observe that combinatorial manifolds are always nice.

Let $\left(\sigma_{0}, \sigma_{1}, \ldots, \sigma_{l}\right)$ be an ordering of the facets of a pure simplicial $d$-complex $K$, and let $D_{i}=\bigcup_{0<j<i} \sigma_{j}$ denote the union of the first $i$ facets. We call the ordering $\left(\sigma_{0}, \sigma_{1}, \ldots, \sigma_{l}\right)$ a shelling of $K$ if $D_{i-1} \cap \sigma_{i}$ is a pure simplicial $(d-1)$-complex for $1 \leq i \leq l$. If $K$ is the boundary complex of a simplicial $(d+1)$-polytope, then $K$ admits a shelling order which can be computed efficiently; see Ziegler [27, Chapter 8].

A simplicial complex obtained from a shellable complex by stellar subdivision of a face is again shellable, a shellable sphere or ball is a combinatorial sphere or ball, and for $1 \leq i \leq l$ the intersection $D_{i-1} \cap \sigma_{i}$ is a combinatorial $(d-1)$-ball (or sphere). A shellable simplicial complex $K$ is a wedge of balls or spheres in general. If $K$ is a manifold, then $D_{i}$ is a combinatorial $d$-ball (or sphere) for $0 \leq i \leq l$, and in particular we have that $D_{i-1} \cap \sigma_{i}, D_{i}$, and hence $K$ are nice. We call a face $f \subset \sigma_{i}$ free if $f \notin D_{i-1}$. In particular the (inclusion) minimal free faces describe all free faces, and they are also called restriction sets in the theory of $h$-vectors of simplicial polytopes.

1.2 The branched cover. The concept of a covering of a space $Y$ by another space $X$ is generalized by Fox [4] to the notion of the branched cover. Here a certain subset $Y_{\text {sing }} \subset Y$ may violate the conditions of a covering map. This allows for a wider application in the construction of topological spaces. It is essential for a satisfactory theory of (branched) coverings to make certain connectivity assumption for $X$ and $Y$. The spaces mostly considered are Hausdorff, path connected, and locally path connected; see Bredon [3, III.3.1]. Throughout we will restrict our attention to coverings of manifolds, hence they meet the connectivity assumptions in [3].

Consider a continuous map $h: Z \rightarrow Y$, and assume the restriction $h: Z \rightarrow h(Z)$ to be a covering. If $h(Z)$ is dense in $Y$ (and meets certain additional connectivity conditions) then there is a surjective map $p: X \rightarrow Y$ with $Z \subset X$ and $\left.p\right|_{Z}=h$. The map $p$ is called a completion of $h$, and any two completions $p: X \rightarrow Y$ and $p^{\prime}: X^{\prime} \rightarrow Y$ are equivalent in the sense that there exists a homeomorphism $\varphi: X \rightarrow X^{\prime}$ satisfying $p^{\prime} \circ \varphi=p$ and $\left.\varphi\right|_{Z}=\mathrm{Id}$. The map $p: X \rightarrow Y$ obtained this way is a branched cover, and we call the unique minimal subset $Y_{\text {sing }} \subset Y$ such that the restriction of $p$ to the preimage of $Y \backslash Y_{\text {sing }}$ is a covering the branching set of $p$. The restriction of $p$ to $p^{-1}\left(Y \backslash Y_{\text {sing }}\right)$ is called the associated covering of $p$. If $h: Z \rightarrow Y$ is a covering, then $X=Z$, and $p=h$ is a branched cover with empty branching set.

Example 1.1. For $k \geq 2$ consider the map $p_{k}: \mathbb{C} \rightarrow \mathbb{C}: z \mapsto z^{k}$. The restriction $\left.p_{k}\right|_{\mathbb{D}^{2}}$ is a $k$-fold branched cover $\mathbb{D}^{2} \rightarrow \mathbb{D}^{2}$ with the single branch point $\{0\}$.

We define the monodromy homomorphism

$$
\mathfrak{m}_{p}: \pi_{1}\left(Y \backslash Y_{\text {sing }}, y_{0}\right) \rightarrow \operatorname{Sym}\left(p^{-1}\left(y_{0}\right)\right)
$$


of a branched cover for a point $y_{0} \in Y \backslash Y_{\text {sing }}$ as the monodromy homomorphism of the associated covering: If $[\alpha] \in \pi_{1}\left(Y \backslash Y_{\text {sing }}, y_{0}\right)$ is represented by a closed path $\alpha$ based at $y_{0}$, then $\mathfrak{m}_{p}$ maps $[\alpha]$ to the permutation $\left(x_{i} \mapsto \alpha_{i}(1)\right)$, where $\left\{x_{1}, x_{2}, \ldots, x_{k}\right\}=p^{-1}\left(y_{0}\right)$ is the preimage of $y_{0}$ and $\alpha_{i}:[0,1] \rightarrow X$ is the unique lifting of $\alpha$ with $p \circ \alpha_{i}=\alpha$ and $\alpha_{i}(0)=x_{i}$; see Munkres [19, Lemma 79.1] and Seifert \& Threlfall [23, §58]. The monodromy group $\mathfrak{M}_{p}$ is defined as the image of $\mathfrak{m}_{p}$.

Two branched covers $p: X \rightarrow Y$ and $p^{\prime}: X^{\prime} \rightarrow Y^{\prime}$ are equivalent if there are homeomorphisms $\varphi: X \rightarrow X^{\prime}$ and $\psi: Y \rightarrow Y^{\prime}$ with $\psi\left(Y_{\text {sing }}\right)=Y_{\text {sing }}^{\prime}$ such that $p^{\prime} \circ \varphi=\psi \circ p$ holds. The well-known Theorem 1.2 is due to the uniqueness of $Y_{\text {sing }}$, and hence the uniqueness of the associated covering; see Piergallini [20, p. 2].

Theorem 1.2. Let $p: X \rightarrow Y$ be a branched cover of a connected manifold $Y$. Then $p$ is uniquely determined up to equivalence by the branching set $Y_{\operatorname{sing}}$ and the monodromy homeomorphism $\mathfrak{m}_{p}$. In particular, the covering space $X$ is determined up to homeomorphisms.

Let $Y$ be a connected manifold and $Y_{\text {sing }}$ a codimension 2 submanifold, possibly with a finite number of singularities. We call a branched cover $p$ simple if the image $\mathfrak{m}_{p}(m)$ of any meridial loop $m$ around a non-singular point of the branching set is a transposition in $\mathfrak{M}_{p}$. Note that the $k$-fold branched cover $\left.p_{k}\right|_{\mathbb{D}^{2}}: \mathbb{D}^{2} \rightarrow \mathbb{D}^{2}$ presented in Example 1.1 is not simple for $k \geq 3$.

1.3 The partial unfolding. The partial unfolding $\widehat{K}$ of a simplicial complex $K$ first appeared in a paper by Izmestiev \& Joswig [14], with some of the basic notions already developed in Joswig [15]. The partial unfolding is closely related to the complete unfolding, also defined in [14], but we will not discuss the latter. The partial unfolding is a geometric object defined entirely by the combinatorial structure of $K$, and comes along with a canonical projection $p: K \rightarrow K$.

However, the partial unfolding $\widehat{K}$ may not be a simplicial complex. In general $\widehat{K}$ is a pseudo-simplicial complex: Let $\Sigma$ be a collection of pairwise disjoint geometric simplices with simplicial attaching maps for some pairs $(\sigma, \tau) \in \Sigma \times \Sigma$, mapping a subcomplex of $\sigma$ isomorphically to a subcomplex of $\tau$. Identifying the subcomplexes accordingly yields the quotient space $\Sigma / \sim$, which is called a pseudo-simplicial complex if the quotient map $\Sigma \rightarrow \Sigma / \sim$ restricted to any $\sigma \in \Sigma$ is bijective. The last condition ensures that there are no self-identifications within each simplex $\sigma \in \Sigma$.

The group of projectivities. Let $\sigma$ and $\tau$ be neighboring facets of a finite, pure simplicial complex $K$, that is, $\sigma \cap \tau$ is a ridge. Then there is exactly one vertex in $\sigma$ which is not a vertex of $\tau$ and vice versa, hence a natural bijection $\langle\sigma, \tau\rangle$ between the vertex sets of $\sigma$ and $\tau$ is given by

$$
\langle\sigma, \tau\rangle: V(\sigma) \rightarrow V(\tau): v \mapsto\left\{\begin{array}{lll}
v & \text { if } & v \in \sigma \cap \tau \\
\tau \backslash \sigma & \text { if } & v=\sigma \backslash \tau .
\end{array}\right.
$$

The bijection $\langle\sigma, \tau\rangle$ is called the perspectivity from $\sigma$ to $\tau$. 


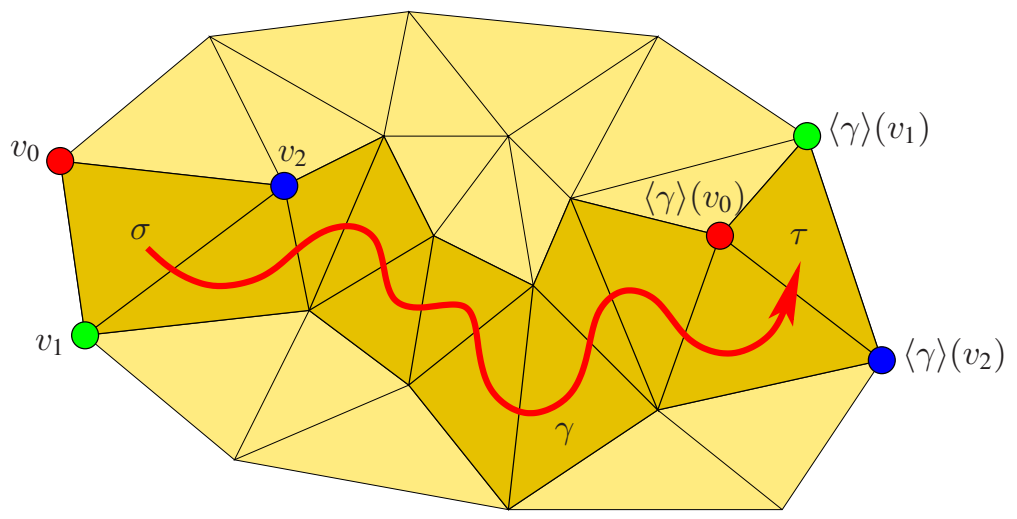

Figure 1. A projectivity from $\sigma$ to $\tau$ along the facet path $\gamma$.

A facet path in $K$ is a sequence $\gamma=\left(\sigma_{0}, \sigma_{1}, \ldots, \sigma_{k}\right)$ of facets such that the corresponding nodes in the dual graph $\Gamma^{*}(K)$ form a path, that is, $\sigma_{i} \cap \sigma_{i+1}$ is a ridge for all $0 \leq i<k$; see Figure 1. Now the projectivity $\langle\gamma\rangle$ along $\gamma$ is defined as the composition of perspectivities $\left\langle\sigma_{i}, \sigma_{i+1}\right\rangle$, thus $\langle\gamma\rangle$ maps $V\left(\sigma_{0}\right)$ to $V\left(\sigma_{k}\right)$ bijectively via

$$
\langle\gamma\rangle=\left\langle\sigma_{k-1}, \sigma_{k}\right\rangle \circ \cdots \circ\left\langle\sigma_{1}, \sigma_{2}\right\rangle \circ\left\langle\sigma_{0}, \sigma_{1}\right\rangle .
$$

We write $\gamma \delta=\left(\sigma_{0}, \sigma_{1}, \ldots, \sigma_{k}, \sigma_{k+1}, \ldots, \sigma_{k+l}\right)$ for the concatenation of two facet paths $\gamma=\left(\sigma_{0}, \sigma_{1}, \ldots, \sigma_{k}\right)$ and $\delta=\left(\sigma_{k}, \sigma_{k+1}, \ldots, \sigma_{k+l}\right)$, denote by $\gamma^{-}=\left(\sigma_{k}, \sigma_{k-1}\right.$, $\left.\ldots, \sigma_{0}\right)$ the inverse path of $\gamma$, and we call $\gamma$ a closed facet path based at $\sigma_{0}$ if $\sigma_{0}=\sigma_{k}$. The set of closed facet paths based at $\sigma_{0}$ together with the concatenation forms a group, and a closed facet path $\gamma$ based at $\sigma_{0}$ acts on the set $V\left(\sigma_{0}\right)$ via $\gamma \cdot v=\langle\gamma\rangle(v)$ for $v \in V\left(\sigma_{0}\right)$. Via this action we obtain the group of projectivities $\Pi\left(K, \sigma_{0}\right)$ given by all permutations $\langle\gamma\rangle$ of $V\left(\sigma_{0}\right)$. The group of projectivities is a subgroup of the symmetric group $\operatorname{Sym}\left(V\left(\sigma_{0}\right)\right)$ on the vertices of $\sigma_{0}$.

The projectivities along null-homotopic closed facet paths based at $\sigma_{0}$ generate the subgroup $\Pi_{0}\left(K, \sigma_{0}\right)$ of $\Pi\left(K, \sigma_{0}\right)$, which is called the reduced group of projectivities. Finally, if $K$ is strongly connected then $\Pi\left(K, \sigma_{0}\right)$ and $\Pi\left(K, \sigma_{0}^{\prime}\right)$, respectively $\Pi_{0}\left(K, \sigma_{0}\right)$ and $\Pi_{0}\left(K, \sigma_{0}^{\prime}\right)$, are isomorphic for any two facets $\sigma_{0}, \sigma_{0}^{\prime} \in K$. In this case we usually omit the base facet in the notation of the (reduced) group of projectivities, and write $\Pi(K)=\Pi\left(K, \sigma_{0}\right)$, respectively $\Pi_{0}(K)=\Pi_{0}\left(K, \sigma_{0}\right)$.

The odd subcomplex. Let $K$ be locally strongly connected; in particular, $K$ is pure. The link of a codimension 2-face $f$ is a graph which is connected since $K$ is locally strongly connected, and $f$ is called even if the link $\operatorname{lk}_{K}(f)$ of $f$ is 2-colorable (i.e. bipartite as a graph), and odd otherwise. We define the odd subcomplex of $K$ as all odd codimension 2 -faces (together with their proper faces), and denote it by $K_{\text {odd }}$ (or sometimes odd $(K)$ ).

Assume that $K$ is pure and admits a $(d+1)$-coloring of its graph $\Gamma(K)$, that is, we assign one color of a set of $d+1$ colors to each vertex of $\Gamma(K)$ such that the two vertices 
of any edge carry different colors. Observe that the $(d+1)$-coloring of $K$ is minimal with respect to the number of colors, and is unique up to renaming the colors if $K$ is strongly connected. Simplicial complexes that are $(d+1)$-colorable are called foldable, since a $(d+1)$-coloring defines a non-degenerate simplicial map of $K$ to the $(d+1)$-simplex. In other places in the literature foldable simplicial complexes are sometimes called balanced.

Lemma 1.3. The odd subcomplex of a foldable simplicial complex $K$ is empty, and the group of projectivities $\Pi\left(K, \sigma_{0}\right)$ is trivial. In particular we have $\langle\gamma\rangle=\langle\delta\rangle$ for any two facet paths $\gamma$ and $\delta$ from $\sigma$ to $\tau$ for any two facets $\sigma, \tau \in K$.

We leave the proof to the reader. As we will see in Theorem 1.4 the odd subcomplex is of interest in particular for its relation to $\Pi_{0}\left(K, \sigma_{0}\right)$ of a nice simplicial complex $K$.

Consider a geometric realization $|K|$ of $K$. To a given facet path $\gamma=\left(\sigma_{0}, \sigma_{1}, \ldots, \sigma_{k}\right)$ in $K$ we associate a (piecewise linear) path $|\gamma|$ in $|K|$ by connecting the barycenter of $\sigma_{i}$ to the barycenters of $\sigma_{i} \cap \sigma_{i-1}$ and $\sigma_{i} \cap \sigma_{i+1}$ by a straight line for $1 \leq i<k$, and connecting the barycenters of $\sigma_{0}$ and $\sigma_{0} \cap \sigma_{1}$, respectively $\sigma_{k}$ and $\sigma_{k} \cap \sigma_{k-1}$. A projectivity around a codimension 2-face $f$ is a projectivity along a facet path $\gamma \delta \gamma^{-}$, where $\delta$ is a closed facet path in $\operatorname{st}_{K}(f)$ (based at some facet $\sigma \in$ st $_{K}(f)$ ) such that $|\gamma|$ is homotopy equivalent to the boundary of a transversal disc around $|f| \subset \mid$ st $_{K}(f) \mid$, and $\gamma$ is a facet path from $\sigma_{0}$ to $\sigma$. The path $\gamma \delta \gamma^{-}$is null-homotopic since $K$ is locally strongly simply connected.

Theorem 1.4 (Izmestiev \& Joswig [14, Theorem 3.2.2]). The reduced group of projectivities $\Pi_{0}\left(K, \sigma_{0}\right)$ of a nice simplicial complex $K$ is generated by projectivities around the odd codimension 2-faces. In particular, $\Pi_{0}\left(K, \sigma_{0}\right)$ is generated by transpositions.

The fundamental group $\pi_{1}\left(|K| \backslash\left|K_{\text {odd }}\right|, y_{0}\right)$ of a nice simplicial complex $K$ is generated by paths $|\gamma|$, where $\gamma$ is a closed facet path based at $\sigma_{0}$, and $y_{0}$ is the barycenter of $\sigma_{0}$; see [14, Proposition A.2.1]. Furthermore, due to Theorem 1.4 we have the group homomorphism

$$
\mathfrak{h}_{K}: \pi_{1}\left(|K| \backslash\left|K_{\text {odd }}\right|, y_{0}\right) \rightarrow \Pi\left(K, \sigma_{0}\right):[|\gamma|] \mapsto\langle\gamma\rangle,
$$

where $[|\gamma|]$ is the homotopy class of the path $|\gamma|$ corresponding to a facet path $\gamma$.

The partial unfolding. Let $K$ be a pure simplicial $d$-complex and set $\Sigma$ as the set of all pairs $(|\sigma|, v)$, where $\sigma \in K$ is a facet and $v \in \sigma$ is a vertex. Thus each pair $(|\sigma|, v) \in \Sigma$ is a copy of the geometric simplex $|\sigma|$ labeled by one of its vertices. For neighboring facets $\sigma$ and $\tau$ of $K$ we define the equivalence relation $\sim$ by attaching $(|\sigma|, v) \in \Sigma$ and $(|\tau|, w) \in \Sigma$ along their common ridge $|\sigma \cap \tau|$ if $\langle\sigma, \tau\rangle(v)=w$ holds. Now the partial unfolding $\widehat{K}$ is defined as the quotient space $\widehat{K}=\Sigma / \sim$. The projection $p: \widehat{K} \rightarrow K$ is given by the factorization of the map $\Sigma \rightarrow K:(|\sigma|, v) \mapsto \sigma$; see Figure 2 .

The partial unfolding of a strongly connected simplicial complex is not strongly connected in general. We denote by $\widehat{K}_{(|\sigma|, v)}$ the connected component containing the labeled facet $(|\sigma|, v)$. Clearly, $\widehat{K}_{(|\sigma|, v)}=\widehat{K}_{(|\tau|, w)}$ holds if and only if there is a facet path $\gamma$ from $\sigma$ to $\tau$ in $K$ with $\langle\gamma\rangle(v)=w$. It follows that the connected components of $\widehat{K}$ correspond to the orbits of the action of $\Pi\left(K, \sigma_{0}\right)$ on $V\left(\sigma_{0}\right)$. Note that each connected component of 

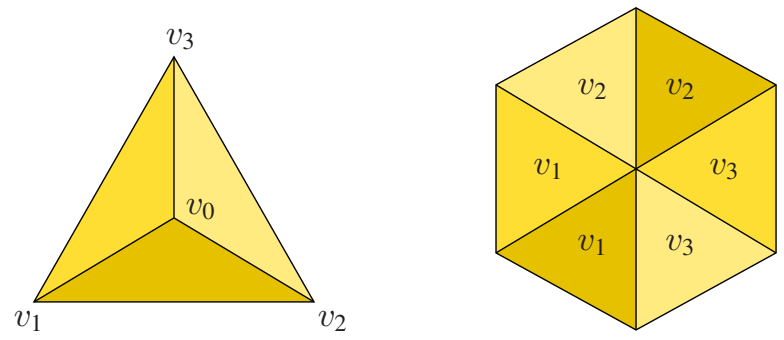

Figure 2. The starred triangle and its partial unfolding. The complex on the right is the non-trivial connected component of the partial unfolding, indicated by the labelling of the facets by the vertices $v_{1}, v_{2}$, and $v_{3}$. The second connected component is a copy of the starred triangle with all facets labelled $v_{0}$; see also Example 1.1 for $k=2$.

the partial unfolding is strongly connected and locally strongly connected [24, Satz 3.2.2]. Therefore we do not distinguish between connected and strongly connected components of the partial unfolding.

The problem that the partial unfolding $\widehat{K}$ may not be a simplicial complex can be addressed in several ways. Izmestiev \& Joswig [14] suggest barycentric subdivision of $\widehat{K}$, or anti-prismatic subdivision of $K$. A more efficient solution (with respect to the size of the resulting triangulations) is given in [24].

1.4 The partial unfolding as a branched cover. As preliminaries to this section we state two theorems by Fox [4] and Izmestiev \& Joswig [14]. Together they imply that under the "usual connectivity assumptions" the partial unfolding of a simplicial complex is indeed a branched cover as suggested in the heading of this subsection.

Theorem 1.5 (Izmestiev \& Joswig [14, Theorem 3.3.2]). Let $K$ be a nice simplicial complex. Then the restriction of $p: \widehat{K} \rightarrow K$ to the preimage of the complement of the odd subcomplex is a simple covering.

Theorem 1.6 (Fox [4, p. 251]; Izmestiev \& Joswig [14, Proposition 4.1.2]). Let $J$ and $K$ be nice simplicial complexes and let $f: J \rightarrow K$ be a simplicial map. Then the map $f$ is a simplicial branched cover if and only if

$$
\operatorname{codim} K_{\text {sing }} \geq 2
$$

Since the partial unfolding of a nice simplicial complex is nice, Corollary 1.7 follows immediately.

Corollary 1.7. Let $K$ be a nice simplicial complex. The projection $p: \widehat{K} \rightarrow K$ is a simple branched cover with the odd subcomplex $K_{\mathrm{odd}}$ as its branching set. 
For the rest of this section let $K$ be a nice simplicial complex and let $y_{0}$ be the barycenter of a fixed facet $\sigma_{0} \in K$. The projection $p: \widehat{K} \rightarrow K$ is a branched cover with $K_{\text {sing }}=K_{\text {odd }}$ by Corollary 1.7, and Izmestiev \& Joswig [14] proved that there is a bijection $\imath: p^{-1}\left(y_{0}\right) \rightarrow V\left(\sigma_{0}\right)$ that induces a group isomorphism $\imath_{*}: \operatorname{Sym}\left(p^{-1}\left(y_{0}\right)\right) \rightarrow$ $\operatorname{Sym}\left(V\left(\sigma_{0}\right)\right)$ such that the following Diagram (1) commutes.

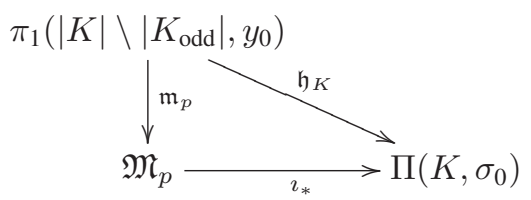

Let $r: X \rightarrow Y$ be a branched cover and assume that there is a homeomorphism of pairs $\varphi:\left(Y, Y_{\text {sing }}\right) \rightarrow\left(|K|,\left|K_{\text {odd }}\right|\right)$, that is, $\varphi: Y \rightarrow|K|$ is a homeomorphism with $\varphi\left(Y_{\text {sing }}\right)=\left|K_{\text {odd }}\right|$. Then Theorem 1.8 gives sufficient conditions for $p: \widehat{K} \rightarrow K$ and $r: X \rightarrow Y$ to be equivalent branched covers. It is the key tool in the proof of the main Theorem 2.1 in Section 2.

Theorem 1.8. Let $K$ be a nice simplicial complex and let $r: X \rightarrow Y$ be a (simple) branched cover. Further assume that there is a homeomorphism of pairs $\varphi:\left(Y, Y_{\text {sing }}\right) \rightarrow$ $\left(|K|,\left|K_{\text {odd }}\right|\right)$, and let $y_{0} \in Y$ be a point such that $\varphi\left(y_{0}\right)$ is the barycenter of $\left|\sigma_{0}\right|$ for some facet $\sigma_{0} \in K$. The branched covers $p: \widehat{K} \rightarrow K$ and $r: X \rightarrow Y$ are equivalent if there is a bijection $\iota: r^{-1}\left(y_{0}\right) \rightarrow V\left(\sigma_{0}\right)$ that induces a group isomorphism $\iota_{*}: \mathfrak{M}_{r} \rightarrow \Pi\left(K, \sigma_{0}\right)$ such that the diagram

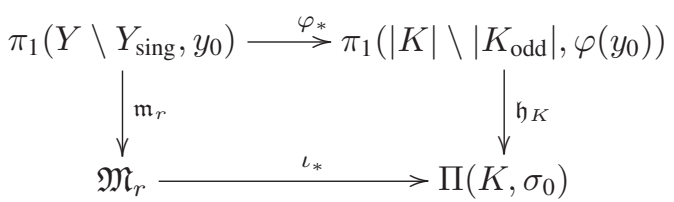

commutes. In particular, we have $\widehat{K} \cong X$.

Proof. Corollary 1.7 ensures that $p: \widehat{K} \rightarrow K$ is indeed a branched cover, and commutativity of Diagram (1) and Diagram (2) proves commutativity of their composition:

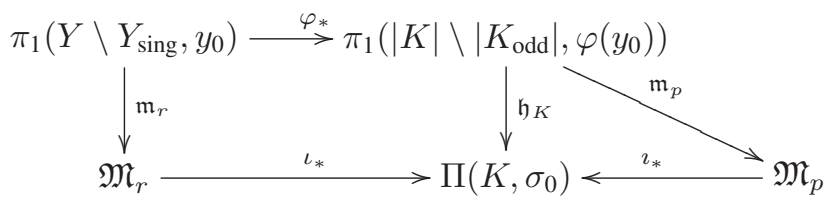

Theorem 1.2 completes the proof.

\section{Constructing branched covers}

Throughout this section let $r: X \rightarrow \mathbb{S}^{d}$ be a branched cover of the $d$-sphere with branching set $F$. The main objective is to give a large class of branched covers $r$, such that there 
is a combinatorial sphere $S$ with $p: \widehat{S} \rightarrow S$ equivalent to $r$ as a branched cover. In particular this implies the existence of a homeomorphism of pairs $\varphi:\left(\mathbb{S}^{d}, F\right) \rightarrow\left(|S|,\left|S_{\text {odd }}\right|\right)$. Note that by the nature of the partial unfolding and the projection $p: \widehat{S} \rightarrow S$, any branched cover $r$ equivalent to $p$ has to be simple and $(d+1)$-fold. A theorem similar to Theorem 2.1 may easily be formulated for branched covers of $d$-balls.

Recall that we associate to a facet path $\gamma$ in $S$ the (realized) path $|\gamma|$ in $|S|$, and that the square brackets denote the homotopy class of a closed path. Thus we write $\mathfrak{m}_{r}\left(\left[\varphi^{-1}(|\gamma|)\right]\right)$ for the image of an element in $\pi_{1}\left(\mathbb{S}^{d} \backslash F, y_{0}\right)$ represented by the closed path $\varphi^{-1}(|\gamma|)$, which in turn is obtained from a closed facet path $\gamma$ based at some facet $\sigma_{0} \in S$ with barycenter $\varphi\left(y_{0}\right)$ by first considering its realization $|\gamma|$ and then its preimage under $\varphi$.

Theorem 2.1. For $d \geq 2$ let $r: X \rightarrow \mathbb{S}^{d}$ be a $(d+1)$-fold, simple branched cover of the $d$-sphere, and assume that the branching set $F$ of $r$ can be embedded via a homeomorphism $\varphi: \mathbb{S}^{d} \rightarrow\left|S^{\prime}\right|$ into the codimension 2-skeleton of a shellable simplicial d-sphere $S^{\prime}$. Then there is a shellable simplicial d-sphere $S$ such that $p: \widehat{S} \rightarrow S$ is a branched cover equivalent to $r$. Furthermore, the $d$-sphere $S$ can be obtained from $S^{\prime}$ by a finite series of stellar subdivision of edges. If $S^{\prime}$ is the boundary of a simplicial $(d+1)$-polytope, then also $S$ is the boundary of a simplicial $(d+1)$-polytope.

To make the proof of Theorem 2.1 more digestible we first give the (algorithmical) back-bone of the proof and defer some of the more technical aspects to the Lemmas 2.2, 2.3, and 2.4. Fix a point $y_{0} \in \mathbb{S}^{d} \backslash F$; we may assume $\varphi\left(y_{0}\right)$ to be the barycenter of some facet $\sigma_{0} \in S^{\prime}$ and $\left|\sigma_{0}\right| \cap \varphi(F)=\emptyset$ to hold. Further fix a bijection $\imath$ between the preimage $\left\{x_{0}, x_{1}, \ldots, x_{d}\right\}=r^{-1}\left(y_{0}\right)$ of $y_{0}$ and the vertices of $\sigma_{0}$, and color the vertices of $\sigma_{0}$ via $\imath$ by the elements in $r^{-1}\left(y_{0}\right)$.

The $d$-sphere $S$ is constructed in a finite series $\left(S^{\prime}=S_{0}, S_{1}, \ldots, S_{l}=S\right)$ of shellable $d$-spheres, and each $d$-sphere $S_{i}$ comes with a shelling of its facet with marked beginning $\left(\sigma_{i, 0}, \sigma_{i, 1}, \ldots, \sigma_{i, l_{i}}\right)$. The complex $S_{i+1}$ is obtained from $S_{i}$ by (possibly) subdividing $\sigma_{i, l_{i}+1}$ in a finite series of stellar subdivisions of edges not contained in any $\sigma_{i, j}$ for $0 \leq$ $j \leq l_{i}$. Thus we may choose the shelling of $S_{i+1}$ such that it extends $\left(\sigma_{i, 0}, \sigma_{i, 1}, \ldots, \sigma_{i, l_{i}}\right)$ and we denote the marked beginning of the shelling of $S_{i}$ simply by $\left(\sigma_{0}, \sigma_{1}, \ldots, \sigma_{l_{i}}\right)$.

Let $D_{i}=\bigcup_{0 \leq j \leq l_{i}} \sigma_{j}$. Then the main idea of the proof of Theorem 2.1 is to construct $S_{i}$ such that the branched covers $r: X \rightarrow \mathbb{S}^{d}$ (restricted to $\varphi^{-1}\left(\left|D_{i}\right|\right)$ ) and $\widehat{D_{i}} \rightarrow D_{i}$ are equivalent. To this end we prove that $\varphi$ restricted to $\varphi^{-1}\left(\left|D_{i}\right|\right)$ is a homeomorphism of pairs $\left(\varphi^{-1}\left(\left|D_{i}\right|\right), F \cap \varphi^{-1}\left(\left|D_{i}\right|\right)\right) \rightarrow\left(\left|D_{i}\right|,\left|\operatorname{odd}\left(D_{i}\right)\right|\right)$ and that the following Diagram (3) commutes; see Figure 3.

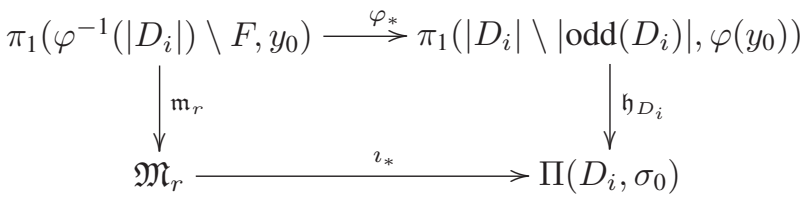

Commutativity of Diagram (3) is obtained by ensuring that for each closed facet path $\gamma$ in $D_{i}$ (which is not a facet path in $D_{i-1}$ ) the projectivity $\langle\gamma\rangle$ acts on $V\left(\sigma_{0}\right)$ as $m_{r}\left(\left[\varphi^{-1}(|\gamma|)\right]\right)$ acts on $r^{-1}\left(y_{0}\right)$. 


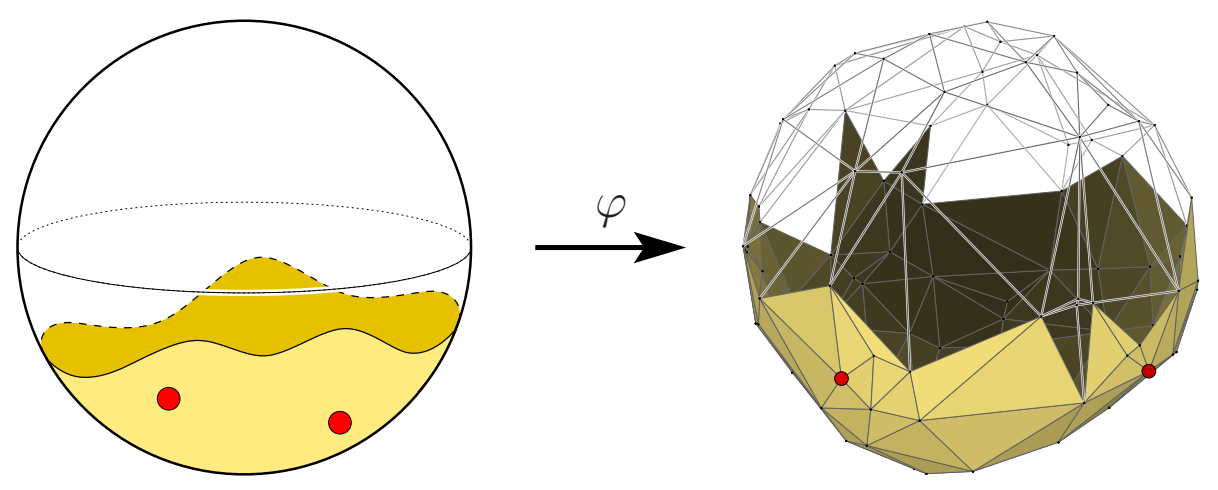

Figure 3. The base space of the branched cover $r: X \rightarrow \mathbb{S}^{2}$ (left) and a polytopal 2-sphere $S_{i}$ with marked beginning $\left(\sigma_{j}\right)_{0 \leq j \leq l_{i}}$ of a shelling (right). On the left the preimage of $D_{i}=\bigcup_{0<j<l_{i}} \sigma_{j}$ under the homomorphism $\varphi: \mathbb{S}^{2} \rightarrow\left|S_{i}\right|$ is shaded and the branching set is marked. The odd subcomplex of $D_{i}$ is marked on the right. The branched covers $r: X \rightarrow \mathbb{S}^{2}$ (restricted to $\varphi^{-1}\left(\left|D_{i}\right|\right)$ ) and $\widehat{D_{i}} \rightarrow D_{i}$ are equivalent.

The pair $\left(S_{i+1},\left(\sigma_{j}\right)_{0 \leq j \leq l_{i+1}}\right)$ is constructed from the pair $\left(S_{i},\left(\sigma_{j}\right)_{0 \leq j \leq l_{i}}\right)$ as follows. Let $\sigma=\sigma_{l_{i}+1}$ be the first facet in the shelling of $S_{i}$ not contained in $D_{i}$, let $\gamma$ be a facet path in $D_{i} \cup \sigma$ from $\sigma_{0}$ to $\sigma$, and let $f \subset \sigma$ be a face. Further let $H_{f, \gamma}$ be the subgroup of $\mathfrak{M}_{r}$ which is induced via $m_{r}$ by all elements of $\pi_{1}\left(\mathbb{S}^{d} \backslash F, y_{0}\right)$ of the form $\left[\varphi^{-1}\left(\left|\gamma \delta \gamma^{-1}\right|\right)\right]$, where $\delta$ is any closed facet path in $\operatorname{st}_{S_{i}}(f)$ based at $\sigma$. The subgroup $H_{f, \gamma}$ has at least $\operatorname{dim}(f)+1$ trivial orbits, namely, the orbits corresponding to the vertices of $f$, and for $g \subset f$ we have that the set of trivial orbits of $H_{f, \gamma}$ contains the trivial orbits of $H_{g, \gamma}$. We consider the following three case:

(i) The intersection $\sigma \cap D_{i}$ is a ridge $f$. Let $\gamma$ be a facet path in $D_{i} \cup \sigma$ from $\sigma_{0}$ to $\sigma$, and color $\sigma$ (and hence $f$ ) by the coloring induced along $\gamma$ by the fixed coloring of $\sigma_{0}$. Now keep the coloring of $f$, but change the color of the remaining vertex $v=\sigma \backslash f$ to any trivial orbit of $H_{v, \gamma}$; see Figure 4 (right).

(ii) The intersection $\sigma \cap D_{i}$ equals two ridges $f \cup v$ and $f \cup w$ with a common codimension 2-face $f$. Let $\sigma_{v} \in D_{i}$ be the facet intersecting $\sigma$ in $f \cup v$, let $\sigma_{w} \in D_{i}$ be the facet intersecting $\sigma$ in $f \cup w$, and choose facet paths $\gamma$ from $\sigma_{0}$ to $\sigma_{v}$ in $D_{i}$ and $\delta$ from $\sigma_{v}$ to $\sigma_{w}$ in $\operatorname{st}_{D_{i}}(f)$. The fixed coloring of $\sigma_{0}$ induces along $\gamma$, respectively $\gamma \delta$, colorings on $f \cup v$ and $f \cup w$, and the colorings coincide on $f$. Now we change the color of $w$ according to $\mathfrak{m}_{r}\left(\left[\varphi^{-1}\left(\left|\gamma \delta\left(\sigma_{w}, \sigma, \sigma_{v}\right) \gamma^{-1}\right|\right)\right]\right)$, which is either a transposition (changing the color of $w$ ) or the identity; see Figure 4 (left).

(iii) Otherwise set $S_{i+1}=S_{i}$ and let $\left(\sigma_{0}, \sigma_{1}, \ldots, \sigma_{l_{i}}, \sigma\right)$ be the marked beginning of a shelling of $S_{i+1}$.

We obtained a (possibly inconsistent) coloring of the vertices of $\sigma$ in the Cases (i) and (ii). Note that the coloring of $\sigma$ induces a consistent coloring on $D_{i} \cap \sigma$, and that there is at most one conflicting edge $\{v, w\}$, that is, $v$ and $w$ are colored the same. A consistently colored subdivision of $\sigma$ is constructed in at most $d-1$ subdivisions of $\sigma$ with exactly 


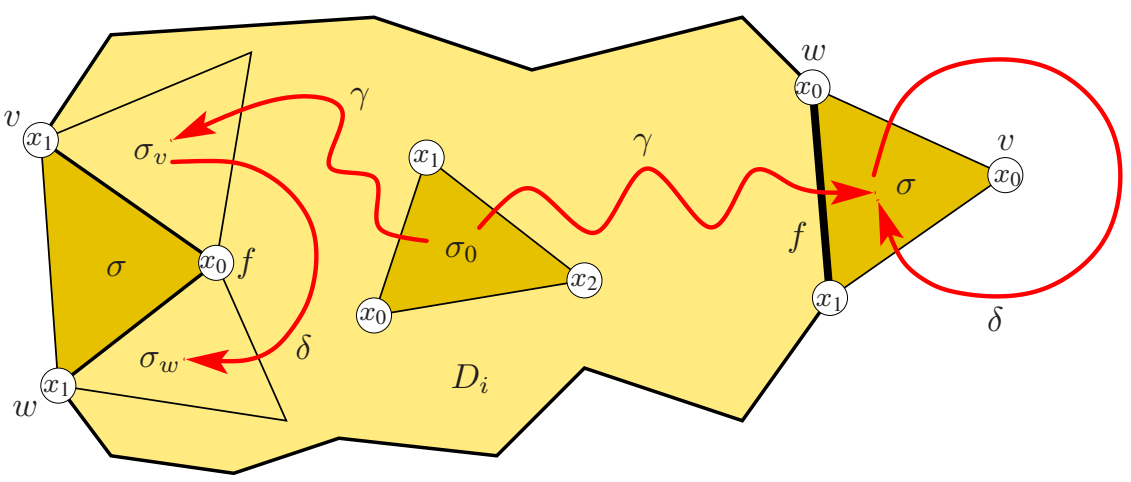

Figure 4. Case (i): The 2-ball $D_{i}$ with the facet $\sigma_{0}$ colored via $\imath$ by the preimage $\left\{x_{0}, x_{1}, x_{2}\right\}$ of $y_{0}$ and induced coloring of the ridge $f$ on the right hand side of the figure. The vertex $v$ is colored $x_{0}$ if any element of $\mathfrak{M}_{r}$ corresponding via $m_{r} \circ \varphi^{-1}$ to a facet path of the form $\gamma \delta \gamma^{-1}$ maps $x_{0}$ to itself. Case (ii): The induced coloring of the codimension 2-face $f$ and the vertices $v$ and $w$ on the left. The edge $\{v, w\}$ is subdivided if the facet path $\gamma \delta\left(\sigma_{w}, \sigma, \sigma_{v}\right) \gamma^{-1}$ corresponds via $m_{r} \circ \varphi^{-1}$ to the identity in $\mathfrak{M}_{r}$.

one conflicting edge $e$ each, where each subdivision is obtained from the previous one by stellar subdividing $e$ : Let $f_{e} \subset \sigma$ be the unique minimal face such that $|e| \subset|f|$ holds and denote by $C_{e}$ the set of trivial orbits of $H_{f_{e}, \gamma}$. Now color the new vertex $v_{e}$ with an element of $C_{e}$ which is not the color of any vertex $v_{e^{\prime}}$ subdividing an edge $e^{\prime}$ with $f_{e^{\prime}} \subset f$. Note that $C_{e}$ is the entire preimage $r^{-1}\left(y_{0}\right)$ if $f_{e}$ is a codimension 1-face, and that $C_{e}$ has at least one element distinct from the colors of all $v_{e^{\prime}}$ for $f_{e^{\prime}} \subset f_{e}$. If $C_{e}$ contains the one color $x \in r^{-1}\left(y_{0}\right)$ not used in the coloring of $\sigma$, color $v_{e}$ by $x$ and terminate the subdivision process.
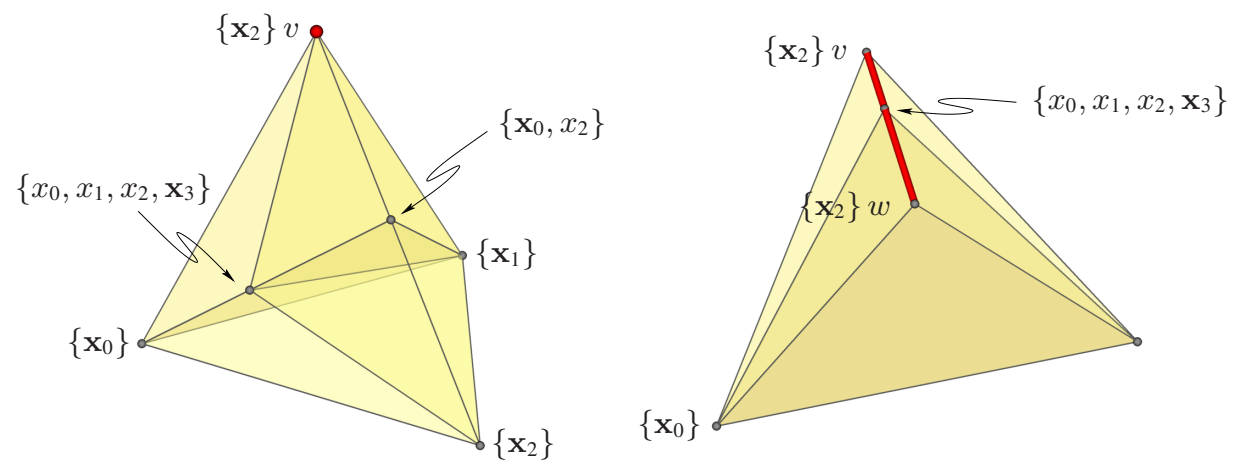

Figure 5. Coloring of the vertices of the refinement of $\sigma$ in Case (i) (on the left) and Case (ii) (on the right). The minimal free face $v$, respectively $\{v, w\}$, is marked. Each vertex $v_{e}$ is labeled by the trivial orbits of $H_{f_{e}, \gamma}$ and the vertex color is printed bold. 
This completes the construction of $S_{i+1}$ in the Cases (i) and (ii), and we define the marked beginning of a shelling of $S_{i+1}$ by $\left(\sigma_{0}, \sigma_{1}, \ldots, \sigma_{l_{i}}\right)$ followed by the facets of the refinement of $\sigma$ in an appropriate order.

It remains to prove that the algorithm described above terminates and that $p: \widehat{S} \rightarrow S$ is a branched cover equivalent to $r: X \rightarrow \mathbb{S}^{d}$. Since $S$ is shellable and hence nice, $p$ is a branched cover by see Corollary 1.7. The following Lemmas 2.2 and 2.3 prove the equivalence of $p$ and $r$, while termination of the construction above is provided by Lemma 2.4 .

Lemma 2.2. The branched covers $p: \widehat{S} \rightarrow S$ and $r: X \rightarrow \mathbb{S}^{d}$ are equivalent.

Proof. In order to show the equivalence of the branched covers $p$ and $r$ we prove by induction that the following holds for $0 \leq i \leq l$ :

(I) For any closed facet path $\gamma$ based at $\sigma_{0}$ in $D_{i}$ we have $\langle\gamma\rangle=\imath_{*} \circ \mathfrak{m}_{r}\left(\left[\varphi^{-1}(|\gamma|)\right]\right)$.

(II) Let $v \in D_{i}$ be a vertex, and let $\gamma$ be a facet path in $D_{i}$ from $\sigma_{0}$ to a facet $\sigma$ containing $v$. Then the color induced on $v$ along $\gamma$ by the fixed coloring of $\sigma_{0}$ is a trivial orbit of $H_{v, \gamma}$.

We remark that (I) implies that $\varphi$ restricted to $\varphi^{-1}\left(\left|D_{i}\right|\right)$ is a homeomorphism of pairs $\left(\varphi^{-1}\left(\left|D_{i}\right|\right), F \cap \varphi^{-1}\left(\left|D_{i}\right|\right)\right) \rightarrow\left(\left|D_{i}\right|,\left|\operatorname{odd}\left(D_{i}\right)\right|\right)$ and that Diagram (3) commutes. Finally, (I) and (II) are met for the pair $\left(S_{0}, D_{0}\right)=\left(S^{\prime}, \sigma_{0}\right)$, and commutativity of Diagram (3) proves the equivalence of $r: X \rightarrow \mathbb{S}^{d}$ and $p: \widehat{S} \rightarrow S$ for $i=l$; see Theorem 1.8.

We show that (I) and (II) hold for the pair $\left(S_{i+1}, D_{i+1}\right)$ provided they hold for the pair $\left(S_{i}, D_{i}\right)$. Recall that we denote the first facet $\sigma_{l_{i}+1}$ of the shelling of $S_{i}$ not contained in $D_{i}$ by $\sigma$. The simplicial complex $D_{i}$ is contractible and hence $\Pi_{0}\left(D_{i}, \sigma_{0}\right)=\Pi\left(D_{i}, \sigma_{0}\right)$ is generated by closed facet paths around (odd) codimension 2-faces by Theorem 1.4. Thus it suffices to verify (I) for closed facet paths around (interior) codimension 2-faces by examining the three Cases (i), (ii), and (iii).

(i) The intersection $\sigma \cap D_{i}$ is a ridge $f$. New interior codimension 2-faces in $D_{i+1}$ arise only in the refinement of $\sigma$, which is foldable by construction. Since $\varphi(F)$ does not intersect the interior of $|\sigma|$, any facet path around a new interior codimension 2-face corresponds to the identity of $\mathfrak{M}_{r}$ and (I) holds by Lemma 1.3.

(ii) The intersection $\sigma \cap D_{i}$ equals two ridges $f \cup v$ and $f \cup w$ with a common codimension 2-face $f$. By induction hypothesis (II) holds for the vertices of $f$ in $D_{i}$ and thus (I) follows for the new interior codimension 2-face $f$ of $D_{i+1}$ by construction. As for any new interior codimension 2-face in the refinement of $\sigma$, (I) holds (as in Case (i)) since the refinement is foldable and $\varphi(F)$ does not intersect the interior of $|\sigma|$.

(iii) Otherwise there is no codimension 2-faces $f \subset \sigma$ with a free corresponding edge $e_{f}=\sigma \backslash f$ and (I) follows from Lemma 2.3.

Having established (I), it suffices to verify (II) for a single facet path $\gamma$ in $D_{i+1}$ from $\sigma_{0}$ to any facet containing a given vertex $v$. Thus (II) holds by choice of color for any vertex added to $D_{i}$ in the construction of the pair $\left(S_{i+1}, D_{i+1}\right)$.

Lemma 2.3. If $f \in \sigma$ is a codimension 2-face with a non-free corresponding edge $e_{f}=$ $\sigma \backslash f$, then (I) holds for any closed facet path based at $\sigma_{0}$ around $f$ in $D_{i+1}$. 
Proof. Let $\gamma \delta \gamma^{-1}$ be a closed facet path based at $\sigma_{0}$ around $f$ in $D_{i+1}$, where $\delta$ is a closed path around $f$ in $\operatorname{st}_{D_{i+1}}(f)$. Since $\{v, w\}=e_{f}$ is a non-free edge, there is a facet path $\delta^{\prime}$ in $D_{i}$ with $\left|\delta^{\prime}\right|$ homotopy equivalent to $\left|\left\{f_{e}, f \cup v, f \cup w\right\}\right|$ in $\left|D_{i}\right| \backslash\left|\operatorname{odd}\left(D_{i}\right)\right|$, and we assume $\delta$ and $\delta^{\prime}$ to have the same orientation; see Figure 6. Note that the complex $\left\{f_{e}, f \cup v, f \cup w\right\}$ itself is homotopy equivalent to $\mathbb{S}^{1}$.

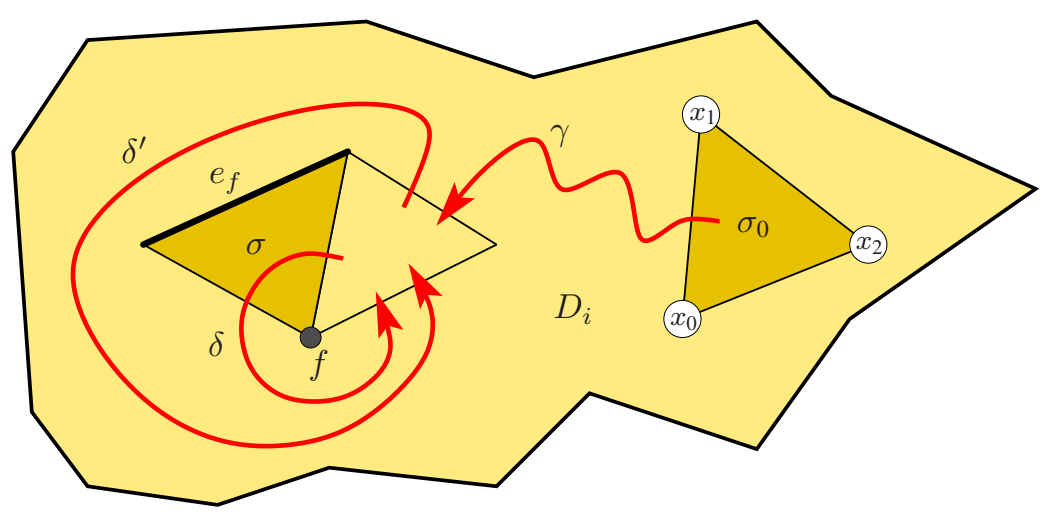

Figure 6. Case (iii): The paths $\gamma, \delta$, and $\delta^{\prime}$ if the corresponding edge $e_{f}$ of a codimension 2-face $f$ is non-free.

Without loss of generality let $m_{r}\left(\left[\varphi^{-1}\left(\left|\gamma \delta \gamma^{-1}\right|\right)\right]\right)$ either be the identity or the transposition $\left(x_{0}, x_{1}\right) \in \mathfrak{M}_{r}$. Each transposition $\left(x_{i}, x_{j}\right)$, for $i \neq j$, appears at most once in the (unique) reduced representation of the element $a=m_{r}\left(\left[\varphi^{-1}(|A|)\right]\right) \in \mathfrak{M}_{r}$ corresponding to the facet path $A=\gamma \delta^{\prime} \gamma^{-1}$, since $A$ is composed from facet paths around codimension 2-faces of $\sigma$. Let $b=m_{r}\left(\left[\varphi^{-1}(|B|)\right]\right) \in \mathfrak{M}_{r}$ denote the element corresponding to the facet path $B=\gamma \delta^{\prime} \delta^{-1} \gamma^{-1}$, then $a=\left(x_{0}, x_{1}\right) \circ b$ holds if and only if $\left(x_{0}, x_{1}\right)$ is in the reduced representation of $a$, and we have $a=b$ otherwise. Since (I) holds for $D_{i}$ and hence in particular for the facet path $A$, and with

$$
A=\gamma \delta^{\prime} \gamma^{-1}=\gamma \delta^{\prime} \delta^{-1} \gamma^{-1} \gamma \delta \gamma^{-1}=B \gamma \delta \gamma^{-1},
$$

we conclude that the projectivity along $\gamma \delta \gamma^{-1}$ is the identity on the vertices of $\sigma_{0}$ if and only if $\gamma \delta \gamma^{-1}$ corresponds via $\mathfrak{m}_{r} \circ \varphi^{-1}$ to the identity in $\mathfrak{M}_{r}$, and exchanges exactly the vertices colored $x_{0}$ and $x_{1}$ otherwise.

The following Lemma 2.4 proves termination of the construction of the shellable $d$ sphere $S$ and completes the proof of Theorem 2.1.

Lemma 2.4. The shellable $d$-sphere $S$ is obtained by finitely many stellar subdivisions of edges.

Proof. We prove that no facet will be subdivided more than a finite number of times in the construction of $S$. The facet $\sigma_{l_{i}+i}$ is subdivided at most $d-1$ times in the construction 
$S_{i+1}$ from $S_{i}$, and no facet in $D_{i}$ is subdivided. The refinement of $\sigma_{l_{i}+1}$ is added to $D_{i}$ to define $D_{i+1}$ and no facet in the refinement will be subdivided any further.

Problems may accrue since subdividing $\sigma_{l_{i}+1}$ results in subdividing other facets (not in $D_{i}$ ) intersecting $\sigma_{l_{i}+1}$, and each facet of the refinement of an intersecting facet appears in the shelling, yet is not in $D_{i+1}$. Thus a facet might get subdivided over and over again.

For a face $f \in S^{\prime}$ let $L_{f, i} \subset S_{i}$ denote the refinement of $f$ in $S_{i}$. Without loss of generality we may assume that the facets of the refinement $L_{\sigma, i}$ of any facet $\sigma \in S^{\prime}$ appear consecutively in the shelling order of $S_{i}$. Let $\sigma \in S^{\prime}$ be a fixed facet and let $i_{0}$ be the number such that $S_{i_{0}}$ is the $d$-sphere with $\sigma_{l_{i_{0}}+1}$ is the facet of $L_{\sigma, i_{0}}$ appearing first in the shelling order, that is, $S_{i_{0}+1}$ is constructed by adding (a refinement) of the first facet of $L_{\sigma, i_{0}}$ to $D_{i_{0}}$. Thus we obtain an induced coloring of the boundary vertices of $L_{\sigma, i_{0}}$ which is consistent on $D_{i_{0}} \cap L_{\sigma, i_{0}}$ by construction. Since $\varphi(F)$ does not intersect the interior of $\left|L_{\sigma, i_{0}}\right|$ and by Lemma 1.3, it remains to prove that this coloring of $D_{i_{0}} \cap L_{\sigma, i_{0}}$ extends to a foldable refinement of $L_{\sigma, i_{0}}$ obtained via a finite series of stellar subdivisions.

Observe that each facet of $L_{\sigma, i_{0}}$ is the cone over a $(d-1)$-simplex in the boundary of $L_{\sigma, i_{0}}$ and that $L_{\sigma, i_{0}}$ has no interior vertices: This is obviously true for $L_{\sigma, 0}=\sigma$. For $1 \leq i \leq i_{0}$ let cone $(f)$ be a facet of $L_{\sigma, i-1}$ with $f$ is a boundary $(d-1)$-simplex. Now if cone $(f)$ is subdivided via stellarly subdividing an edge $e \in f$, both facets replacing cone $(f)$ are cones over boundary $(d-1)$-simplices which in turn are obtained from $f$ by replacing one vertex of $e$ by the new vertex subdividing $e$.

We strengthen the statement above and claim that each facet of $L_{\sigma, i_{0}}$ is the cone over a $(d-1)$-simplex in $D_{i_{0}} \cap L_{\sigma, i_{0}}$. To this end note the trivial fact that if $e \in L_{g, i}$ is an edge of the subdivision of a boundary $k$-face $g \in \sigma$ and if $\left\{f_{j}\right\}_{1 \leq j \leq d-k}$ are the boundary $(d-1)$-faces of $\sigma$ with $g=\bigcap_{1 \leq j \leq d-k} f_{j}$, then there is a $(d-1)$-simplex in each $L_{f_{j}, i}$ containing $e$. Thus if for some $i<i_{0}$ an edge $e$ is subdivided when adding the simplex $\sigma_{l_{i}+1}$ to $D_{i}$ which intersects $L_{\sigma, i}$ in a low dimensional face, then at least one of the boundary $(d-1)$-simplices of $L_{\sigma, i}$ containing $e$ will be added to $D_{i^{\prime}} \cap L_{\sigma, i^{\prime}}$ at some point $i<i^{\prime} \leq i_{0}$.

Returning to the consistent coloring of $D_{i_{0}} \cap L_{\sigma, i_{0}}$ we conclude that all vertices of $L_{\sigma, i_{0}}$ are colored since there are no interior vertices, and that each facet cone $(f)$ of $L_{\sigma, i_{0}}$ has at most one conflicting edge since the boundary $(d-1)$-simplex $f \subset D_{i_{0}} \cap L_{\sigma, i_{0}}$ is consistently colored. Hence $\mathrm{st}_{L_{\sigma, i_{0}}}(e)$ of a conflicting edge $e$ does not contain any other conflicting edges and we consider st $L_{\sigma, i_{0}}(e)$ independently.

Now st $L_{\sigma, i_{0}}(e)$ is subdivided only finitely many times since $H_{v, \gamma}$ is trivial for any new vertex $v$ (except for finitely many vertices in the boundary of $\left|\mathrm{st}_{L_{\sigma, i_{0}}}(e)\right|$ ) and hence the construction (Case (i) and (ii)) induces a linear order on the colors used to color the new vertices.

Remark 2.5. It appears as if the shellable $d$-sphere $S$ may be constructed along a spanning tree of the dual graph $\Gamma^{*}\left(S^{\prime}\right)$ instead of a shelling, though the construction would become substantially more complicated. Using a spanning tree of $\Gamma^{*}\left(S^{\prime}\right)$ would eliminate the somehow (to the theory of branched covers) alien concept of a shelling, and would allow for more general base spaces, e.g. PL $d$-manifolds. 

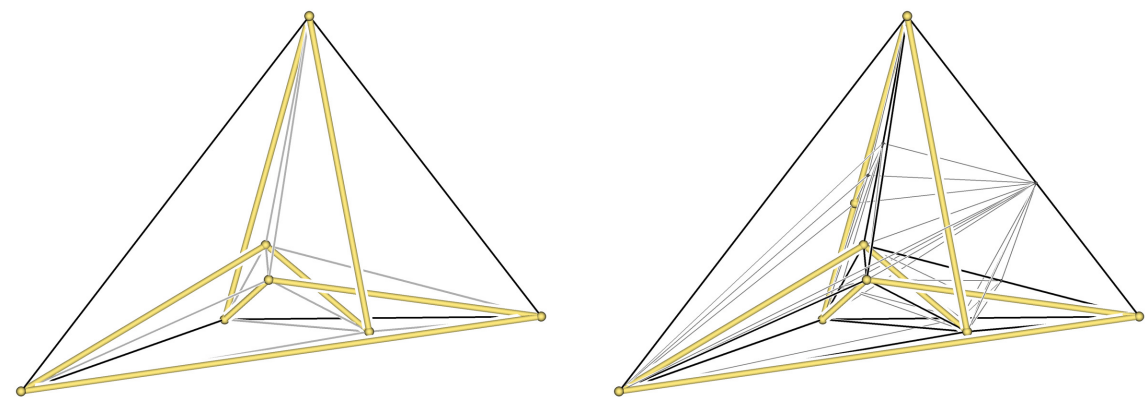

Figure 7. Constructing the trefoil as odd subcomplex of a 3-sphere $S$ with $\Pi(S)$ isomorphic to the symmetric group on three elements. On the left the Schlegel diagram of the cyclic 4-polytope $C_{4,7}$ on seven vertices with the trefoil embedded in the 1-skeleton. On the right $S$ as a subdivision of the Schlegel diagram after stellarly subdividing eight edges of $C_{4,7}$. The odd subcomplex is marked and the $f$-vector of $S$ reads $(15,63,96,48)$; watch [25].

Applying Theorem 2.1 to the results of Hilden [8] and Montesinos [17], Piergallini [21], and Iori \& Piergallini [11] we obtain the following three corollaries.

Corollary 2.6. Let $d=2$ or $d=3$. For every closed oriented $d$-manifold $M$ there is a polytopal d-sphere $S$ such that one of the connected components $\widehat{S}$ of the partial unfolding of $S$ is a combinatorial d-manifold homeomorphic to $M$. The projection $\widehat{S} \rightarrow S$ is a simple $d$-fold branched cover branched over finitely many points for $d=2$, respectively a link for $d=3$.

Corollary 2.7. For every closed oriented PL 4-manifold $M$ there is a polytopal 4-sphere $S$ such that one of the connected components $\widehat{S}$ of the partial unfolding of $S$ is a combinatorial 4-manifold PL-homeomorphic to $M$. The projection $\widehat{S} \rightarrow S$ is a simple 4-fold branched cover branched over a PL-surface with a finite number of cusp and node singularities.

Corollary 2.8. For every closed oriented PL 4-manifold $M$ there is a polytopal 4-sphere $S$ such that the partial unfolding $\widehat{S}$ of $S$ is a combinatorial 4-manifold PL-homeomorphic to $M$. The projection $\widehat{S} \rightarrow S$ is a simple 5-fold branched cover branched over a locally flat PL-surface.

A weaker version of Corollary 2.6 was already established by Izmestiev \& Joswig [14] and later by Hilden, Montesinos, Tejada \& Toro [9]. A weaker version of Corollary 2.7 can be found in [26].

Stellar subdivision of an edge $e \in S$ of a combinatorial $d$-manifold $S$ changes the parity of the codimension 2-faces in $\mathrm{lk}_{S}(e)$. Since the link of an edge of $S$ is a (combinatorial) $(d-2)$-sphere, we obtain the following Corollary 2.9. A topological proof for arbitrary simple branched covers is available by Izmestiev [13]. 
Corollary 2.9. The branching set of a branched cover $r: X \rightarrow \mathbb{S}^{d}$ as described in Theorem 2.1 is the symmetric difference of finitely many $(d-2)$-spheres.

We conclude this section by a remark and a conjecture as to which branched covers $r: X \rightarrow \mathbb{S}^{d}$ may be obtained via the method presented above. In other words, which branching sets can be embedded via a homeomorphism $\varphi: \mathbb{S}^{d} \rightarrow\left|S^{\prime}\right|$ into the codimension 2-skeleton of a shellable simplicial $d$-sphere $S^{\prime}$.

Remark 2.10. For $d \geq 6$ there are branching sets non-embedable into the codimension 2-skeleton of a shellable simplicial $d$-sphere: Freedman \& Quinn [5] constructed a 4manifold which does not have a triangulation as a combinatorial manifold. In fact, there are 4-manifolds which cannot be triangulated at all [16, p. 9].

The branching set of a branched cover $r: X \rightarrow \mathbb{S}^{d}$ for $d \leq 5$ is at most 3-dimensional and since there is no difference between PL and non-PL topology up to dimension three, we conjecture the following.

Conjecture 2.11. For $d \leq 5$ every branched cover $r: X \rightarrow \mathbb{S}^{d}$ can be obtained via the partial unfolding of some polytopal $d$-sphere.

\section{Extending triangulations}

A first assault on how to extend triangulation and coloring is by Goodman \& Onishi [7], who proved that a 4-colorable triangulation of the 2-sphere may be extended to a 4-colorable triangulation of the 3-ball. Their result was improved independently by Izmestiev [12] and [24] to arbitrary dimensions. Here we generalize the construction to arbitrary simplicial complexes with $k$-colored subcomplexes.

Theorem 3.1. Given a simplicial $d$-complex $K$ and a $k$-colored induced subcomplex $L$, then there is a finite series of stellar subdivisions of edges such that the resulting simplicial complex $K^{\prime}$ has a $\max \{k, d+1\}$-coloring, $K^{\prime}$ contains $L$ as an induced subcomplex, and the $\max \{k, d+1\}$-coloring of $K^{\prime}$ induces the original $k$-coloring on $L$.

Proof. We may assume $K$ to be pure. Let $K_{0}=K$ and assign 0 to all vertices not in $L$. For $1 \leq i \leq d$ we obtain the simplicial complex $K_{i}$ from $K_{i-1}$ by stellarly subdividing all conflicting edges with both vertices colored $i-1$ in an arbitrary order. The new vertices are colored $i$. We prove by induction that for $0 \leq j \leq i-1$ and each facet $\sigma \in K_{i}$ there is exactly one vertex $v_{j} \in \sigma$ colored $j$. The assumption holds for $K_{0}$ and completes the proof for $K^{\prime}=K_{d}$. Note that since $L$ is properly colored, no edges in $L$ are subdivided and $L$ is an induced subcomplex of any $K_{i}$ for $0 \leq i \leq d$.

To prove the induction hypothesis for $K_{i}$, we again use an inductive argument: Let $\sigma$ be a facet of a subdivision of $K_{i-1}$ produced in the making of $K_{i}$. Assume that each color less than $i-1$ appears exactly once in $\sigma$, and let $l \geq 2$ be the number of $(i-1)$-colored vertices of $\sigma$. This assumption clearly holds for any facet of $K_{i-1}$ for some $l \leq d-i+2$. After subdividing an $(i-1)$-colored conflicting edge of $\sigma$ and assigning the color $i$ to the 
new vertex, each of the two new facets has $l-1$ vertices colored $i-1$, and each color less than $i-1$ appears exactly once. Thus any facet of $K_{i-1}$ has to be subdivided into at most $2^{d-i+1}$ simplices in order for $K_{i}$ to meet the induction hypothesis.

Izmestiev gives a result similar to Theorem 3.1 in [12], but the following Remark 3.2 points out the advantage of using only stellar subdivisions of edges.

Remark 3.2. Since only stellar subdivisions of edges are used to construct $K^{\prime}$ from $K$, all properties invariant under these subdivisions are preserved, e.g. polytopality, regularity, shellability, and others. In the case that $L$ is not induced, stellarly subdivide all edges $\{v, w\} \in K \backslash L$ with $v, w \in L$. In order to obtain a small triangulation, one can try to (greedily) $(d+1)$-color a (large) foldable subcomplex first.

Corollary 3.3. The odd subcomplex of a closed combinatorial d-manifold is the symmetric difference of finitely many $(d-2)$-spheres.

Corollary 3.4. Given a $k$-colored simplicial $(d-1)$-sphere $S$, then there is a simplicial $d$-ball $D$ with boundary equal to $S$ such that there is a $\max \{k, d+1\}$-coloring of $D$ which induces the original $k$-coloring on $S$. The d-ball $D$ is obtained from $\operatorname{cone}(S)$ by a finite series of stellar subdivision of edges. In particular $D$ is a combinatorial d-ball if $S$ is a combinatorial $(d-1)$-sphere, shellable if $S$ is shellable, and regular if $S$ is polytopal; see Figure 8.
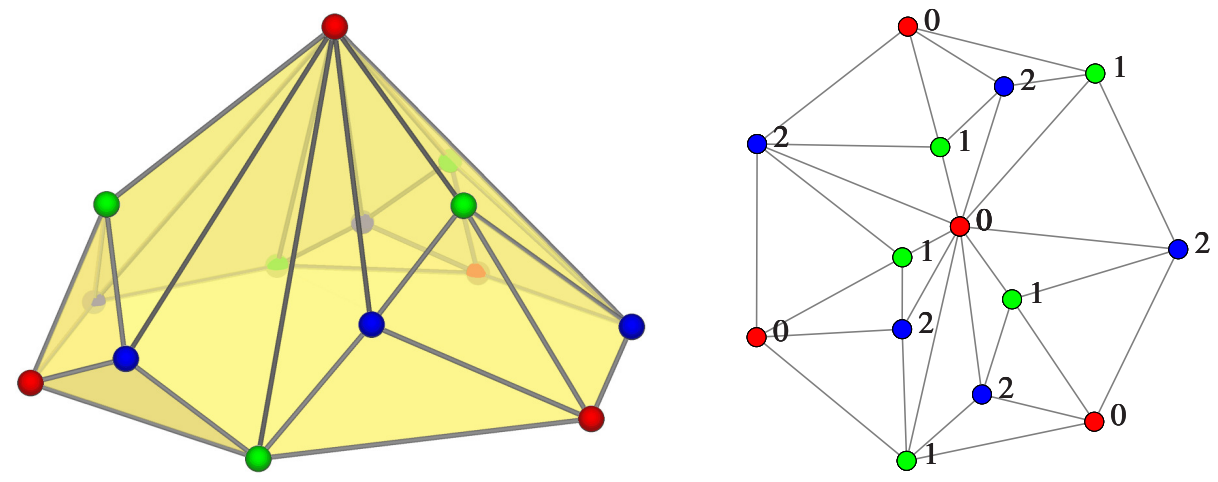

Figure 8. Convex hull of the extended triangulation of a 3-colored 7-gon and its Schlegel diagram.

Remark 3.5. Similar results as Corollary 3.4 may easily be obtained for partial triangulations of a CW-complex and relative handle-body decompositions of a PL-manifold (with boundary). 
The partial unfoldings of two homeomorphic simplicial complexes $K$ and $K^{\prime}$ need not to be homeomorphic in general. We present a notion of equivalence of simplicial complexes which agrees with their unfolding behavior, that is, we give sufficient criteria such that if $p: \widehat{K} \rightarrow K$ and $p^{\prime}: \widehat{K^{\prime}} \rightarrow K^{\prime}$ are branched covers, then $p$ and $p^{\prime}$ are equivalent.

Assume $K$ and $K^{\prime}$ to be strongly connected and that the odd subcomplexes $K_{\text {odd }}$ and $K_{\text {odd }}^{\prime}$ are equivalent, that is, there is a homeomorphism of pairs $\varphi:\left(|K|,\left|K_{\text {odd }}\right|\right) \rightarrow$ $\left(\left|K^{\prime}\right|,\left|K_{\text {odd }}^{\prime}\right|\right)$. Let $\sigma_{0} \in K$ be a facet, and $y_{0}$ the barycenter of $\sigma_{0}$, and assume that the image $y_{0}^{\prime}=\varphi\left(y_{0}\right)$ is the barycenter of $\left|\sigma_{0}^{\prime}\right|$ for some facet $\sigma_{0}^{\prime} \in K^{\prime}$. Now $K$ and $K^{\prime}$ are color equivalent if there is a bijection $\psi: V\left(\sigma_{0}\right) \rightarrow V\left(\sigma_{0}^{\prime}\right)$, such that

$$
\psi_{*} \circ \mathfrak{h}_{K}=\mathfrak{h}_{K^{\prime}} \circ \varphi_{*}
$$

holds, where the maps $\varphi_{*}: \pi_{1}\left(|K| \backslash\left|K_{\text {odd }}\right|, y_{0}\right) \rightarrow \pi_{1}\left(\left|K^{\prime}\right| \backslash\left|K_{\text {odd }}^{\prime}\right|, y_{0}^{\prime}\right)$ and $\psi_{*}:$ $\operatorname{Sym}\left(V\left(\sigma_{0}\right)\right) \rightarrow \operatorname{Sym}\left(V\left(\sigma_{0}^{\prime}\right)\right)$ are the group isomorphisms induced by $\varphi$ and $\psi$, respectively.

Observe that this is indeed an equivalence relation. The name "color equivalent" suggests that the pairs $\left(K, K_{\text {odd }}\right)$ and $\left(K^{\prime}, K_{\text {odd }}^{\prime}\right)$ are equivalent, and that the "colorings" of $K_{\text {odd }}$ and $K_{\text {odd }}^{\prime}$ by the $\Pi(K)$-action, respectively $\Pi\left(K^{\prime}\right)$-action, of projectivities around odd faces are equivalent. Lemma 3.6 justifies this name.

Lemma 3.6. Let $K$ and $K^{\prime}$ be color equivalent nice simplicial complexes. Then the branched covers $p: \widehat{K} \rightarrow K$ and $p^{\prime}: \widehat{K^{\prime}} \rightarrow K^{\prime}$ are equivalent.

Proof. With the notation of Equation (4) we have that

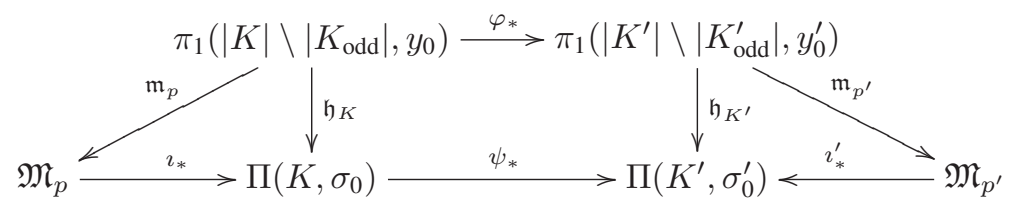

commutes, since Diagram (1) commutes and Equation (4) holds. Theorem 1.2 completes the proof.

Proposition 3.7. For every strongly connected simplicial complex $K$ there is a simplicial complex $K^{\prime}$, obtained from a foldable simplicial complex via a finite series of stellar subdivision of edges, such that $K$ and $K^{\prime}$ are color equivalent.

Theorem 2.1 proves Proposition 3.7 above for shellable spheres. We will not prove the general case and only give a sketch of the construction for general $K$.

Let $L$ be a foldable simplicial complex obtained from $K$ via a finite series of stellar subdivisions according to Theorem 3.1, that is, there is a series $\left(K=K_{0}, K_{1}, \ldots, K_{l}=\right.$ $L)$ where $K_{i}$ is obtained from $K_{i-1}$ by stellarly subdividing a single edge $e_{i-1} \in K_{i-1}$. The idea is to reverse the effect of the stellar subdivisions by subdividing each edge $e$ a second time in the reversed order, since stellarly subdividing $e$ twice yields the antiprismatic subdivision of $e$ (which does not alter the color equivalence class). 
We construct $K^{\prime}$ from $L$ inductively in a series $\left(L=L_{l}, L_{l-1}, \ldots, L_{0}=K^{\prime}\right)$ of simplicial complexes, where $L_{i}$ is obtained from $L_{i+1}$ by a finite series of stellar subdivisions of edges. The complexes $L_{i}$ and $K_{i}$ are color equivalent: For a facet path $\left(\sigma_{j}^{\prime}\right)_{j \in J}$ in $L_{i}$ associate the facet path $\left(\sigma_{j}\right)_{j \in J}$ in $K_{i}$, where $\sigma_{j}$ is the unique facet such that $\left|\sigma_{j}^{\prime}\right|$ lies in $\left|\sigma_{j}\right|$.

We fix some notation in order to describe the construction of $L_{i}$ from $L_{i+1}$. Subdividing the edge $e_{i} \in K_{i}$ in order to construct $K_{i+1}$ replaces $e_{i}$ by two edges in $K_{i+1}$, and we call one of these two edges $e_{i}^{\prime}$. A facet in $\operatorname{st}_{K_{i+1}}\left(e_{i}^{\prime}\right)$ might get subdivided further in the process of constructing $K_{i+2}, K_{i+3}, \ldots, K_{l}=L_{l}, L_{l-1}, \ldots, L_{i+1}$, and we define $L_{e_{i}^{\prime}}$ as the subcomplex of $L_{i+1}$ which refines $\operatorname{st}_{K_{i+1}}\left(e_{i}^{\prime}\right)$.

Note that $e_{i}^{\prime}$ is an edge of $L_{e_{i}^{\prime}}$, and that $L_{e_{i}^{\prime}}$ and $\operatorname{st}_{K_{i+1}}\left(e_{i}^{\prime}\right)$ are color equivalent. It follows that the group of projectivities of $L_{e_{i}^{\prime}}$ has at least two trivial orbits corresponding to the vertices of $e_{i}^{\prime}$. Now $L_{i}$ is obtained from $L_{i+1}$ by stellarly subdividing all edges with vertices belonging to the same two trivial orbits as the vertices of $e_{i}^{\prime}$.

\section{References}

[1] J. W. Alexander, Note on Riemann spaces. Bull. Amer. Math. Soc. 26 (1920), 370-372. MR1560318 Zbl 47.0529.02

[2] A. Björner, Topological methods. In: Handbook of combinatorics, Vol. 1, 2, 1819-1872, Elsevier, Amsterdam 1995. MR1373690 (96m:52012) Zbl 0851.52016

[3] G. E. Bredon, Topology and geometry. Springer 1997. MR1700700 (2000b:55001) Zbl 0934.55001

[4] R. H. Fox, Covering spaces with singularities. In: A symposium in honor of S. Lefschetz, 243-257, Princeton Univ. Press 1957. MR0123298 (23 \#A626) Zbl 0079.16505

[5] M. H. Freedman, F. Quinn, Topology of 4-manifolds. Princeton Univ. Press 1990. MR1201584 (94b:57021) Zbl 0705.57001

[6] E. Gawrilow, M. Joswig, polymake, version 2.1. with contributions by Rörig, T. and Witte, N., free software, 1997-2004. http://www.math.tu-berlin.de/polymake

[7] J. E. Goodman, H. Onishi, Even triangulations of $S^{3}$ and the coloring of graphs. Trans. Amer. Math. Soc. 246 (1978), 501-510. MR515556 (80a:05092) Zbl 0397.05021

[8] H. M. Hilden, Three-fold branched coverings of $S^{3}$. Amer. J. Math. 98 (1976), 989-997. MR0425968 (54 \#13917) Zbl 0342.57002

[9] M. Hilden, J. M. Montesinos, D. Tejada, M. Toro, Representing 3-manifolds by triangulations of $S^{3}$ : a constructive approach. Rev. Colombiana Mat. 39 (2005), 63-86. MR2218370 (2007c:57029) Zbl 1130.57008

[10] J. F. P. Hudson, Piecewise linear topology. Benjamin, New York 1969. MR0248844 (40 \#2094) Zbl 0189.54507

[11] M. Iori, R. Piergallini, 4-manifolds as covers of the 4-sphere branched over non-singular surfaces. Geom. Topol. 6 (2002), 393-401. MR1914574 (2003f:57007) Zbl 1021.57003

[12] I. Izmestiev, Extension of colorings. European J. Combin. 26 (2005), 779-781. MR2127696 (2005j:05032) Zbl 1066.05065 
[13] I. Izmestiev, Addendum to "Branched coverings, triangulations, and 3-manifolds". Personal communication, 2007.

[14] I. Izmestiev, M. Joswig, Branched coverings, triangulations, and 3-manifolds. Adv. Geom. 3 (2003), 191-225. MR1967999 (2004b:57004) Zbl 1025.57008

[15] M. Joswig, Projectivities in simplicial complexes and colorings of simple polytopes. Math. Z. 240 (2002), 243-259. MR1900311 (2003f:05047) Zbl 1054.05039

[16] F. H. Lutz, Triangulated manifolds with few vertices and vertex-transitive group actions. Verlag Shaker, Aachen 1999. MR1866007 (2002h:57034) Zbl 0977.57030

[17] J. M. Montesinos, Three-manifolds as 3-fold branched covers of $S^{3}$. Quart. J. Math. Oxford Ser. (2) 27 (1976), 85-94. MR0394630 (52 \#15431)

[18] J. M. Montesinos, 4-manifolds, 3-fold covering spaces and ribbons. Trans. Amer. Math. Soc. 245 (1978), 453-467. MR511423 (80k:57001) Zbl 0359.55002

[19] J. R. Munkres, Topology: a first course. Prentice-Hall Inc., Englewood Cliffs, N.J. 1975. MR0464128 (57 \#4063) Zbl 0306.54001

[20] R. Piergallini, Manifolds as branched covers of spheres. In: Proceedings of the Eleventh International Conference of Topology (Trieste, 1993), volume 25, 419-439 (1994), 1993. MR1346337 (96g:57004) Zbl 0861.57004

[21] R. Piergallini, Four-manifolds as 4-fold branched covers of $S^{4}$. Topology 34 (1995), 497-508. MR1341805 (96g:57003) Zbl 0869.57002

[22] C. P. Rourke, B. J. Sanderson, Introduction to piecewise-linear topology. Springer 1972. MR0350744 (50 \#3236) Zbl 0254.57010

[23] H. Seifert, W. Threlfall, Seifert and Threlfall: a textbook of topology, volume 89 of Pure and Applied Mathematics. Academic Press 1980. MR575168 (82b:55001) Zbl 0469.55001

[24] N. Witte, Entfaltung simplizialer Sphären. Diplomarbeit, TU Berlin, 2004. http://www.math.tu-berlin.de/ witte/diplom/

[25] N. Witte, Constructing the trefoil as odd subcomplex of a 3-sphere (movie). 2007. http://www.math.tu-berlin.de/ witte/witte_files/cyc.mov

[26] N. Witte, Foldable triangulations. Dissertation, TU Darmstadt, 2007. http://elib.tu-darmstadt.de/diss/000788

[27] G. M. Ziegler, Lectures on polytopes. Springer 1995. MR1311028 (96a:52011) Zbl 0823.52002

Received 15 August, 2007

N. Witte, Institut für Mathematik, MA 6-2, Algorithmische und Diskrete Mathematik, Technische Universität Berlin, Straße des 17. Juni 136, 10623 Berlin, Germany

Email: witte@math.tu-berlin.de 